\title{
DENTAL CARIES PROGNOSIS BY NEURAL NETWORK COMPUTER TECHNOLOGIES
}

\author{
Oleksandr Udod ${ }^{1}$ \\ Hanna Voronina ${ }^{l}$ \\ annavoronina2812@gmail.com \\ ${ }^{1}$ Department of Dentistry No. 1 \\ Donetsk National Medical University \\ 39 Mashinobudivnikiv ave., Kramatorsk, Donetsk region, Ukraine, 84313
}

\begin{abstract}
Computer technologies are widely implemented in clinical dental practice. The use of computer neural network programs in predicting dental caries as the most common dental disease is quite relevant.

The aim - to study the effectiveness of using the "CariesPro" computer program developed using neural network technologies in the individual prediction of dental caries in persons of all ages.

Materials and methods. We examined 73 persons aged 6-7, 12-15 and 35-44 years, in which the intensity of dental caries was determined taking into account the number of cavities, the hygiene condition of the oral cavity, the structural and functional acid resistance of the enamel of the teeth according to the enamel resistance test and its functional component. The data were added to a neural based computer software program "CariesPro" designed to predict dental caries. After 1 year, a second examination was performed and the dental caries obtained were compared with the individually predicted computer program.

Results. The highest intensity of dental caries was found in persons aged 35-44-6.69 \pm 0.38 , in children $6-7$ and $12-15$ years it was $3.85 \pm 0.27$ and $2.15 \pm 0.24$, respectively $(\mathrm{p}<0.05$ ). After 1 year, the corresponding intensity indices for persons of these age categories were $8.92 \pm 0.52 ; 6.27 \pm 0.35$ and $4.23 \pm 0.2$. The growth rates of caries intensity were, respectively, $2.23 \pm 0.25 ; 2.42 \pm 0.15$ and $2.09 \pm 0.15$. After comparing the re-survey data with the computer-programmed estimate, the probable number of carious cavities was found to be 61 true and 12 false predictions from the entire sample, the prediction accuracy of the constructed and trained neural network was $83.56 \%$.

Conclusion. The "CariesPro" computer program, developed using neural network technologies, allows to predict the number of carious lesions in a year with a probability of $83.56 \%$.
\end{abstract}

Keywords: caries, prognosis, computer technology, neural network.

DOI: $10.21303 / 2504-5679.2019 .001070$

\section{Introduction}

Dental caries is one of the most common dental diseases in the world [1,2]. Numerous epidemiological studies conducted in different countries, including Ukraine, show high prevalence rates. Thus, the prevalence of dental caries among the adult population of our country is sometimes approaching $100 \%$, among children of school age it ranges from $60 \%$ to $90 \%[3,4]$. In this regard, the problem of prevention of this disease in people of all ages remains relevant and requires an adequate and effective solution.

One of the probable ways of such decision can be considered introduction in cariology of the principle of predictability and system of individualized caries preventive measures. Known to date, approaches to the prediction of dental caries are based on the determination of dental hygiene, evaluation of oral fluid properties, multifactor computer analysis of local and common factors leading to the development of the disease, mathematical modelling of cariogenic situation, etc. [5, 6]. However, in such approaches, without taking into account the level of caries, or, conversely, the caries of hard tissues of teeth, it is impossible to predict the individual risk of developing caries in a particular person. In this regard, it seems appropriate to have a predictive direction based on the assessment of caries resistance by structural-functional acid resistance of enamel (SFARE), which depends on the functional state of the pulp and is closely related to centrifugal movement of the dental fluid, especially since the well-known clinical express method of its evaluation - the test of enamel resistance (TER) [7]. The use of clinical evaluation of SFARE for TER in predicting dental caries has shown a sufficiently high predictive value. 
In clinical dental practice for the diagnosis and treatment of diseases, as well as for the maintenance of medical records, modelling and manufacturing of orthopaedic structures, planning aesthetic restorations and orthodontic tooth movement, in dental implantation, etc., computer technologies are widely used $[8,9]$. They are constantly being improved, among which systems, whose work is built on the basis of neural network programming, occupy a special place. Such systems are capable of perceiving and analyzing a large amount of information in a short time, making calculations and providing a highly accurate result. In addition, and importantly, these systems are capable of continuous self-improvement and self-learning, which increases the efficiency of their work $[10,11]$. Neuroscience technologies have not yet been used to determine the individual risk of dental caries and to solve clinical problems in dentistry too.

Aim - to study the effectiveness of using the "CariesPro" computer program developed using neural network technologies in the individual prediction of dental caries in persons of all ages.

\section{Materials and methods}

The study was conducted at the Dental Clinic of the Department of Dentistry No. 1 of the Donetsk National Medical University during 2018-2019. In the study we examined 73 individuals of three age groups, including 26 children aged 6-7 years, 34 children 12-15 years and 13 adults aged 35-44 years. Prior to the survey, informed consent was obtained from the parents of the surveyed children and adult patients. In the course of the dental examination, the intensity of dental caries was determined according to the indexes of cfc (caries, filling, cavity) + cfcex (extracted teeth) or CFcEX, taking into account the number of cavities, the hygiene condition of the oral cavity according to the simplified hygienic index Green-Vermillion OHI-S and structural-functional acid resistance clinical evaluation in points of intensity of staining of enamel microdefect area on vestibular surface of intact upper central incisor after dosed acid etching, deep in which corresponds to its certain structural and functional acid resistance [7]. We also determined the functional component of the structural and functional acid resistance of enamel by the test of enamel resistance ( $\triangle T E R)$, for the detection of which, after the initial evaluation of SFARE, the pulp was stimulated by rubbing into the palatal surface of the same test piece of hypertonic sodium chloride solution for 10 minutes, then re-evaluated the SFARE on the vestibular surface of the incisor and determined the difference between the primary and repeated values in points, which was taken as the SFARE functional component [12].

The dental records of all individuals obtained during the initial examination were recorded in the computer program "CariesPro", which was created on the basis of neural networks using Python high-level programming language NumPay and used to build Pandas, TensorFlow, Keras, Scikit-learn software, Matplotlib, JupyterNotebook [11]. CariesPro's graphical user interface was built using Delphi's high-level programming language.

To use this program comfortably, we used a personal computer with certain hardware and software features (Table 1).

Table 1

"CariesPro" hardware and software

\begin{tabular}{cc}
\hline Hardware & Characteristics \\
\hline Central processor & P-III 700MHz or higher \\
Random access memory & not less than $512 \mathrm{Mb}$ \\
Video card and monitor & resolution at least $1024 \times 768$ pixels; \\
Printer & 16 bit colour depth (High colour mode) and up \\
Operating system & MS Windows XP, Windows 7, Windows 8
\end{tabular}

Admin or user work was possible while using the developed software. During the survey, the user entered patient data, predicted the number of likely carious cavities, entered information into the database, and formed a monthly report on patient admission. The administrator configured the neural network system and user access to the system, downloading data to form a training sample for the neural network. 
After the system was started, it was necessary to be authorized, for this it was necessary to fill in a login and password (Fig. 1).

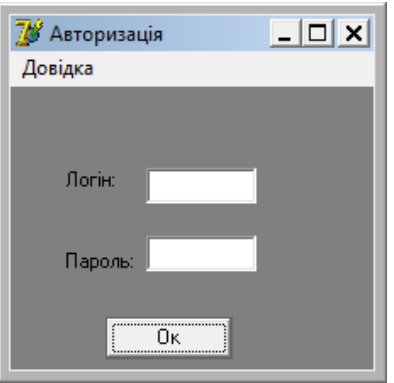

Fig. 1. Authorization form.

If the authorization was performed under the administrator, then the main form of the program for the administrator was opened (Fig. 2). This form contained two tabs "Authorization" and "Download data". In the first section, you could add a new user or edit existing user data. To fill in the new user information, fill in the fields "Last Name First Name", "Login", "Password", and enter his category: "Administrator" or "User" and press "Save" button. Pressing the "Log Out" button could $\log$ off.

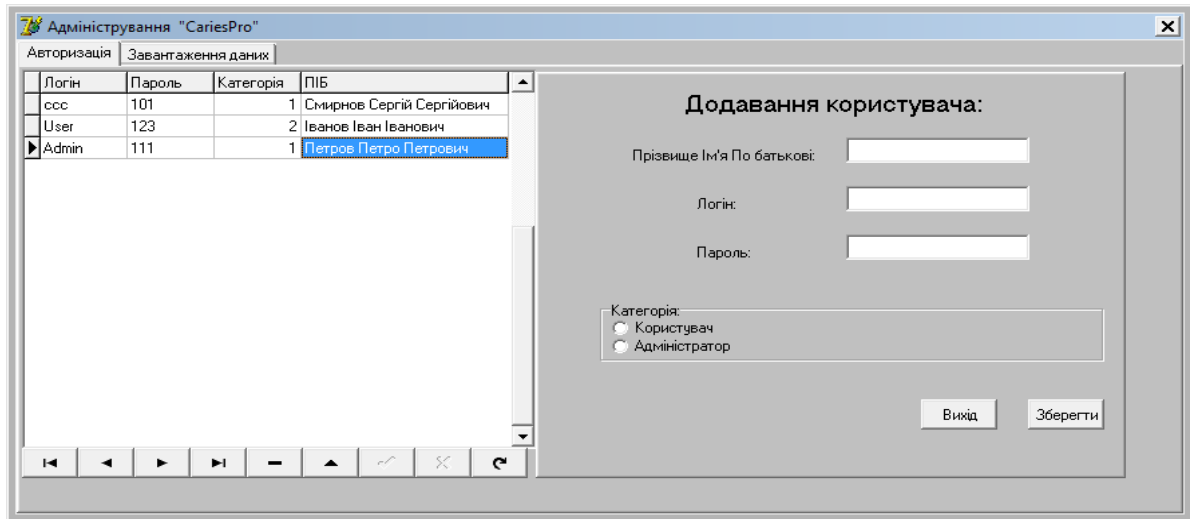

Fig. 2. The main form of the program for the administrator.

In the "Data Upload" window (Fig. 3) we downloaded data for further "learning" of the neural network.

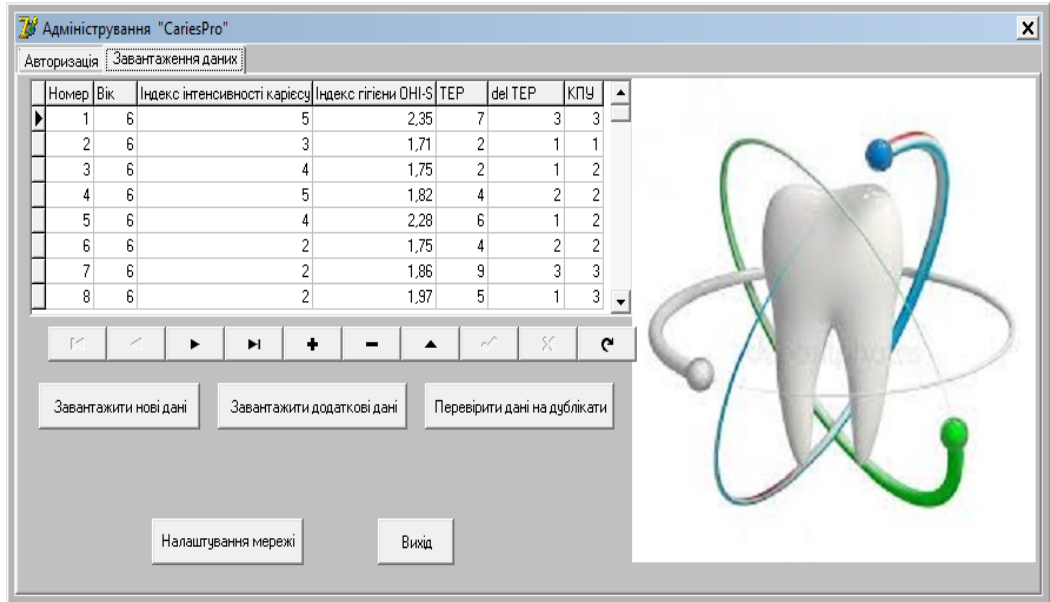

Fig. 3. "Data Upload" window 
If the authorization was performed under the category of user rights, then the main form of the program for the user was opened (Fig. 4).

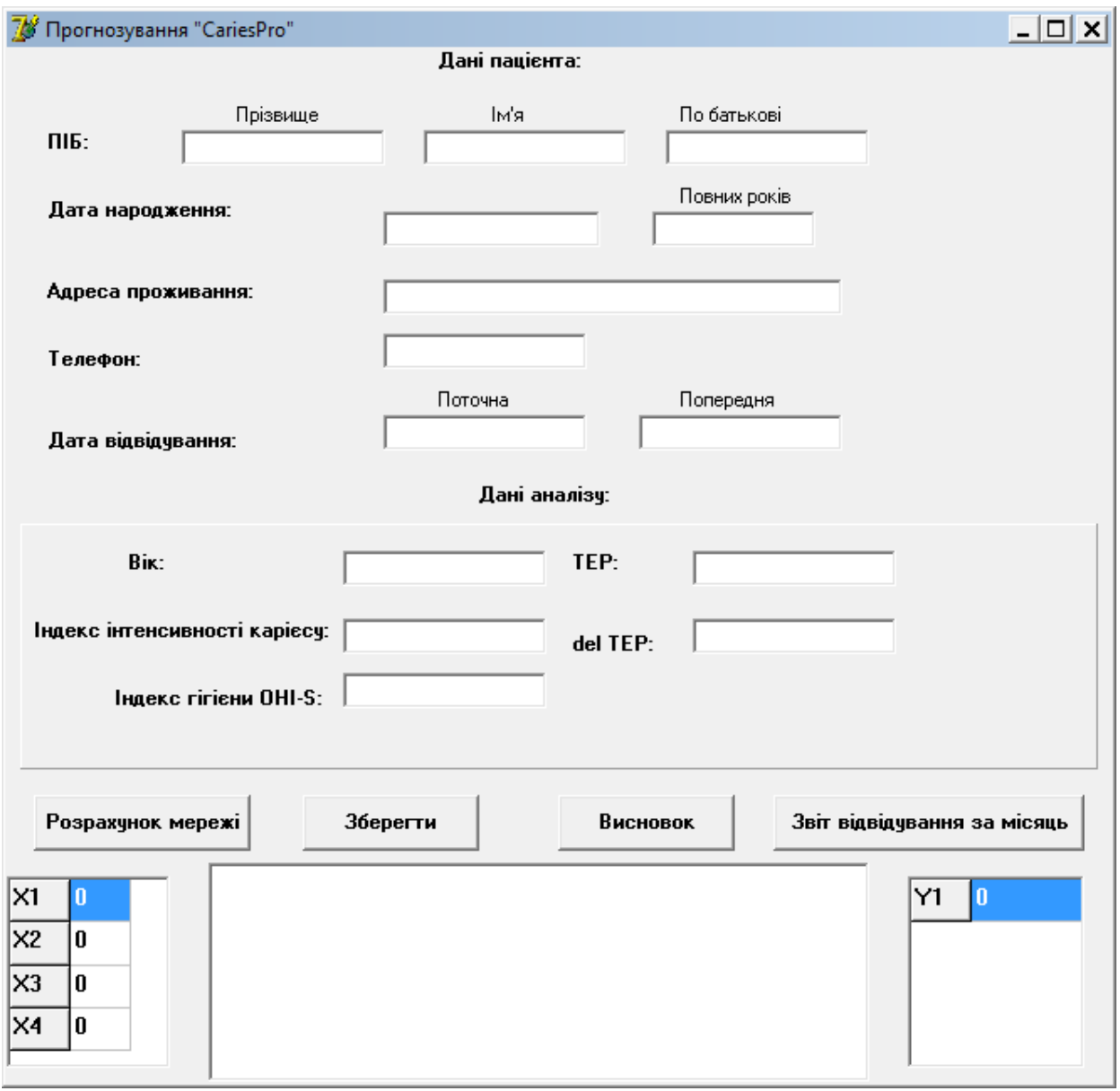

Fig. 4. The main form of the program for the user

While working with the "CariesPro" computer program, each patient completed passport data and entered five dental records that were obtained during the initial examination. In this window, in particular, there were the following buttons:

- "Network calculation" - when this button was pressed, the value of the index of the probable number of carious cavities in the patient was calculated according to the set values in the analysis data;

- "Save" - when this button was pressed the patient's data and estimated values was saved;

- "Monthly visit report" - when this button was pressed, a report on the status of teeth and oral hygiene of patients who visited the dentist last month was generated;

- "Conclusion" - when this button was pressed, a conclusion was drawn up, which contains data on the condition of the teeth and oral hygiene during the current and past examinations of the patient, as well as the results of predicting the probable number of carious cavities based on the obtained dental indicators.

For each of the examined patients, the program formed a general conclusion with the indication of the level (resistance) of tooth enamel to the development of caries and the number of probable carious cavities after 1 year.

After 1 year, a re-examination of all patients was performed, during which each of them again determined the index cfc $+\mathrm{CFcEX}$ or $\mathrm{CFcEX}$, calculated the increase in the intensity of carious lesions by the index $\Delta \mathrm{cfc}+\mathrm{CFcEX}$ or $\triangle \mathrm{CFcEX}$ and compared the obtained rate with the predicted one by computer . 
The results of dental examinations of all patients were recorded in the medical records of the dental patient (form No. 043/o, approved by the Ministry of Health of Ukraine). The preparation of the tables with the primary data and the calculations were performed using the statistical method of linear normalization (NORMALIZATION in Microsoft Excel). To assess the quality of the training sample was determined by the coefficient of repetition and the contradiction coefficient based on the method of statistical evaluation. The results of clinical studies in absolute values were also subjected to statistical processing using the method of variation statistics, the average values of their Excel package.

\section{Results}

During the initial examination of patients, the highest intensity of dental caries on the CFcEX index, taking into account the number of cavities, was found in persons aged 35-44 years, it was $6.69 \pm 0.38$, which, according to WHO criteria, can be attributed to very high level [13]. This index was statistically significant $(\mathrm{p}<0.05)$ higher than the intensity of carious tooth lesions according to the $\mathrm{cfc}+\mathrm{CFcEX}$ index in children aged 6-7 and 12-15 years, in which the corresponding indicators were $3.85 \pm 0.27$ and $2.15 \pm 0.24$. According to the WHO criteria, the average intensity values obtained in children indicate, respectively, medium and low levels of caries. It should be noted that these two indicators also differed statistically significantly $(\mathrm{p}<0.05)$.

The hygienic condition of the oral cavity in the examined patients was unsatisfactory. In this case, the average values of the simplified hygienic index OHI-S in persons of all three age groups were not significantly different from each other $(\mathrm{p}<0.05)$ and, accordingly, in children aged 67 years $1.98 \pm 0.05$ points, in children $12-15$ years $1.87 \pm 0.03$ points, in persons aged $35-44$ years $1.86 \pm 0.05$ points.

The best SFARE tooth score for TER was found in patients of the older age group, that is, in individuals aged $35-44$ years, in which this figure was $4.15 \pm 0.66$ points. Somewhat worse, but with no statistically significant difference $(\mathrm{p}>0.05)$ between themselves and the above were the indicators of structural and functional acid resistance of enamel in children aged 6-7 and 12-15 years, in particular, these values were $4.96 \pm 0.33$ points and $5.00 \pm 0.34$ points, respectively.

In the study of the functional component of structural and functional acid resistance, the highest values were recorded in children aged 6-7 and 12-15 years, in which the indices were almost indistinguishable and were equal to $1.73 \pm 0.15$ points and $1.71 \pm 0.12$ points, respectively $(\mathrm{p}>0.05)$. In patients aged 35-44 years, this indicator was lower by almost 11.5 times $(\mathrm{p}<0.05)$, it was only $0.15 \pm 0.10$ points. Such differences are undoubtedly related to the regularities of the age-related dynamics of the acid resistance of the enamel, the prevalence of its structural component in the elderly, and the gradual and substantial decrease with the age of the role of the functional component [7].

During the re-examination after 1 year, in all examined patients the intensity of dental caries with regard to the number of cavities was statistically significant $(p<0.05)$ increased. The most significant changes were in children, whose age at the initial examination was $12-15$ years, in which this indicator increased almost 2 times as compared to the primary one and made 4.23 \pm 0.24 . Somewhat less, in particular, 1.6 times, there was an increase of the indicator in children aged at the time of the initial examination 6-7 years, in them the intensity of carious lesions of the teeth during the re-examination was $6.27 \pm 0.35$. In individuals aged $35-44$, the caries intensity index increased 1.3 times, this index was $8.92 \pm 0.52$. The results of caries lesion intensity in persons of all ages were statistically significant $(\mathrm{p}<0.05)$.

Indicators of oral hygiene in the surveyed remained at an unsatisfactory level, accounting for children aged 6-7 years $2.12 \pm 0.06$ points, for children aged $12-15$ years $1.98 \pm 0.05$ points, for persons aged $35-44$ years $1.99 \pm 0.07$ points, with no statistically significant difference between them $(\mathrm{p}<0.05)$. However, in children of all ages and adults, statistically significant $(\mathrm{p}<0.05)$ deterioration of the hygiene index did not occur.

The best structural and functional acid resistance of enamel a year later was again found in individuals aged 35-44 years, in particular, this indicator was at the level of $3.92 \pm 0.60$ points. Statistically significant $(\mathrm{p}<0.05)$ worse SFARE of teeth was in children aged 6-7 and $12-15$ years, in 
which its average values were $5.73 \pm 0.37$ points and $5.21 \pm 0.33$ points, respectively. Compared with the primary examination data, a statistically significant difference $(\mathrm{p}<0.05)$ was found only with respect to the SFARE tooth indices in children aged 6-7 years.

Obtained in the survey after 1 year, the indices of increase in the intensity of dental caries by index $\triangle \mathrm{cfc}+\mathrm{CFcEX}$ or $\triangle \mathrm{CFcEX}$, taking into account the number of cavities in the surveyed persons of different age were not statistically different $(\mathrm{p}<0.05)$, they were $6-7$ in children $2.42 \pm 0.15$ years, $2.09 \pm 0.15$ children $12-15$ years, $2.23 \pm 0.25$ persons $35-44$ years old. According to the computer projection, these figures in the surveyed persons after 1 year should be, respectively, $2.27 \pm 0.13$; $1.94 \pm 0.13$ and $2.15 \pm 0.22$. Thus, obtained in the clinical study and calculated on the computer program, the corresponding indicators had no statistically significant differences $(p<0.05)$. It should be noted that the indicators of the increase in the intensity of caries after 1 year were based on the results of comparative analysis of clinical examination and individual forecasts for the computer neural network program "CariesPro". These indices were used to assess the accuracy and effectiveness of predicting carious lesions in the surveyed individuals.

Overall, when comparing patient re-examination data with a computer-programmed prognosis, 61 correct and 12 false predictions from the entire sample were found to be correct, meaning an $83.56 \%$ prediction accuracy of the constructed and trained neural network.

\section{Discussion}

Therefore, there are a sufficient number of ways to predict dental caries, including the involvement of computer programs that can predict the occurrence of this disease in children and adults with varying degrees of likelihood. Most of the available computer programs take into account a wide variety of factors, not always purely clinical, that is, they are multifactorial. Among them is the well-known "Cariogram" program, quite popular in the last decade, but too laborious [5].

Recently, a multifactor computer program, PreViser, has been proposed to predict the development of dental diseases, including dental caries, periodontal disease and oral cancer, which analyzes, in total, eleven factors [6]. This program, in addition to dental and somatic data, also takes into account socio-demographic and behavioural factors, as well as microbiological and oral fluid factors. Determining some of these indicators requires laboratory testing, additional equipment, and other physicians' examinations, resulting in significant financial and time-consuming costs. The result of the program is displayed in a multi-colored chart, which is a bright and significant motivation for patients, but it does not contain information about the number of possible dental caries in a certain time.

Prediction of dental caries with the help of the computer program "CariesPro" avoids the above disadvantages. For its application, it is necessary to identify only five exclusively clinical indicators that can be established during a single dental examination, so that additional examinations and visits are not required. In addition, the program takes into account the exact figures for the condition of the teeth and oral hygiene, which are specific to the individual. The final indicator of working with the CariesPro software is the prediction of the probability of a certain amount of carious cavities after a certain time, which allows to appoint the most individualized caries preventive measures with the setting of the next review deadline.

Study limitations. The study did not include patients 45 years of age or older due to the fact that due to the age-related variability of the structural and functional acid resistance of the enamel, the contribution of the functional component, which should be taken into account in the "CariesPro" computer program, as well as its role in providing caries resistance is minimized. As a result, there may be some restrictions on the use of this computer program to predict dental caries in individuals 45 years of age and older, however, if it is improved by eliminating the mentioned index or introducing one or more new indicators, this limitation may be lifted. Consideration should also be given to the possibility of having caries-preventive measures in the patients examined that affect the effectiveness of prognosis.

Prospects for further research. In the future, it is necessary to conduct studies on the individualized prediction of dental caries using the "CariesPro" computer program over a longer 
period, with an increase in the number of observations and determining the effectiveness of predictions for individuals of different ages. It is also planned to study the effect of caries prevention measures on the effectiveness of forecasting.

\section{Conclusions}

1. Computer program "CariesPro", developed using neural network technologies, allows to determine the individual level of resistance of tooth enamel to caries and to predict in persons of different age the number of caries lesions in a year with a probability of $83.56 \%$, which indicates high efficiency of application of this software product.

2. The small number of clinical indicators that need to be considered in the "CariesPro" computer program, just five of them, can significantly reduce the time and cost of patient screening.

3. The proposed program, through self-study provided by neural network technologies, is capable of perceiving new information regarding the indicators contained therein, which will increase the efficiency of prognosis in the next period of the patient examination.

\section{References}

[1] Kassebaum, N. J., Smith, A. G. C., Bernabé, E., Fleming, T. D., Reynolds, A. E. et. al. (2017). Global, Regional, and National Prevalence, Incidence, and Disability-Adjusted Life Years for Oral Conditions for 195 Countries, 1990-2015: A Systematic Analysis for the Global Burden of Diseases, Injuries, and Risk Factors. Journal of Dental Research, 96 (4), 380-387. doi: http:// doi.org/10.1177/0022034517693566

[2] Frencken, J. E., Sharma, P., Stenhouse, L., Green, D., Laverty, D., Dietrich, T. (2017). Global epidemiology of dental caries and severe periodontitis - a comprehensive review. Journal of Clinical Periodontology, 44, S94-S105. doi: http://doi.org/10.1111/ jcpe. 12677

[3] Bezvushko, E. V., Lahoda, L. S. (2017). Hard dental tissues in children of Lutsk city. Aktualni problemy suchasnoi medytsyny: Visnyk ukrainskoi medychnoi stomatolohichnoi akademii, 17 (2 (58)), 232-235.

[4] Zadorozhna, I. V., Povorozniuk, V. V. (2014). The prevalence and intensity of dental caries among Ukrainian children: findings of clinicoepidemiological surveys. Bil. Suhloby. Khrebet, 1-2 (13-14), 26-29.

[5] Senneby, A., Mejàre, I., Sahlin, N.-E., Svensäter, G., Rohlin, M. (2015). Diagnostic accuracy of different caries risk assessment methods. A systematic review. Journal of Dentistry, 43 (12), 1385-1393. doi: http://doi.org/10.1016/j.jdent.2015.10.011

[6] Zukanović, A. (2013). Caries risk assessment models in caries prediction. Acta Medica Academica, 42 (2), 198-208. doi: http:// doi.org/10.5644/ama2006-124.87

[7] Okushko, V. R. (2005). Osnovy fiziologii zuba. Tiraspol: Izd-vo Pridnestr. un-ta, 240.

[8] Omar, D., Duarte, C. (2018). The application of parameters for comprehensive smile esthetics by digital smile design programs: A review of literature. The Saudi Dental Journal, 30 (1), 7-12. doi: http://doi.org/10.1016/j.sdentj.2017.09.001

[9] Albuha Al-Mussawi, R. M., Farid, F. (2016). Computer-Based Technologies in Dentistry: Types and Applications. Journal of Dentistry, 13 (3), 215-222.

[10] Shah, P., Kendall, F., Khozin, S., Goosen, R., Hu, J., Laramie, J. et. al. (2019). Artificial intelligence and machine learning in clinical development: a translational perspective. Npj Digital Medicine, 2 (1). doi: http://doi.org/10.1038/s41746-019-0148-3

[11] Han, S.-H., Kim, K. W., Kim, S., Youn, Y. C. (2018). Artificial Neural Network: Understanding the Basic Concepts without Mathematics. Dementia and Neurocognitive Disorders, 17 (3), 83-89. doi: http://doi.org/10.12779/dnd.2018.17.3.83

[12] Udod, O. A., Voronina, H. S. (2017). Pat. No. 118523 UA. Sposib vyznachennia funktsionalnoi komponenty strukturno-funktsionalnoi kyslotostiikosti emali zubiv. MPK: G01N 21/91, A61C 17/00. No. u201702237; declareted: 10.03.2017; published: 10.08.2017, Bul. No. 15 .

[13] Oral health surveys: basic methods. 5th ed. World Health Organization (2013). Available at: http://www.who.int/oral_health/ publications/9789241548649/en/ 